\title{
PARTICULARIDADES TÉCNICAS DE BIFACES Y CUCHILLOS DE DORSO NATURAL A LA LUZ DE LAS INVESTIGACIONES EN EL RÎO CORBONES (SEVILLA)
}

\section{TECHNICAL PARTICULARITIES OF HAND AXES AND “NATURALLY BACKED KNIFE" IN THE LIGHT OF NEW RESEARCH AT THE CORBONES RIVER (SEVILLA)}

\author{
por
}

JosÉ JUAN FERNÁNDEZ CARO

\begin{abstract}
RESUMEN Las industrias líticas del Bajo Corbones, afluente del Guadalquivir, se caracterizan por estar realizadas mayoritariamente en cuarcita, materia prima que conforma la casi totalidad de la carga de sus depósitos pleistocenos. Entre sus útiles, los bifaces y cuchillos de dorso natural presentan unas peculiaridades técnicas que los caracterizan, permitiendo en los primeros hacer unas hipótesis de uso y en los segundos reivindicar su carácter de tipo normativo.
\end{abstract}

\begin{abstract}
The lithic industry of the Lower Corbones river, afluent of the Guadalquivir, is characterized by being made principally in quarcite, raw material which composes the practical totality of the load of the deposits. Amongst the tools, the bifaces and couteaux à dos naturel have technical particularities which characterized them, permit us to hypothysize on their use in the first, and revindicate the normative type in the second.
\end{abstract}

Palabras claves Corbones, Guadalquivir, cuarcita, sílex, bifaces y cuchillos de dorso natural.

Key words Corbones, Guadalquivir, quarcite, flint, bifaces and couteaux à dos naturel.

\section{INTRODUCCIÓN}

En el estudio analítico realizado sobre las industrias líticas de cuarenta y cuatro yacimientos en superficie situados en la cuenca del río Corbones, afluente del Guadalquivir por su margen izquierda, hemos detectado unas características peculiares en algunos de los útiles tipológicos, tanto sobre canto como sobre lasca, de las que en este trabajo comentamos las correspondientes a los bifaces y cuchillos de dorso natural. 
Entre los yacimientos citados hemos establecido, desde un punto de vista metodológico, varios grupos en razón de la posición geomorfológica que ocupa. Así, el primer grupo está formado por veintisiete conjuntos que se hallan asociados a afloramientos de la terrazas 2 y 3 del curso bajo del río Corbones (TCB2 y TCB3, cuyo desarrollo discurre entre las T12 y T13 del Guadalquivir -Díaz del Olmo y Vallespí 1992-), proporcionando industrias en cuarcita y sílex morfológica y tecnotipológicamente diferenciadas, siendo las de cuarcita mayoritarias o únicas. El segundo grupo está constituido por tres yacimientos situados en las terrazas 3 y 4 del tramo medio del Corbones (TCM3 y TCM4), correspondiente la primera al paso Pleistoceno Medio-Superior y la segunda al Pleistoceno Superior, con semejantes características a las del grupo anterior (en sus materias primas correspondientes) y donde el sílex es dominante (dos yacimientos) o único. Y el tercero lo forman catorce colecciones detectadas sobre formaciones del Guadalquivir (T6 a T10), cercanos al escarpe de los depósitos de este río colector diseccionados por el curso del Corbones, y por lo tanto en posición dominante sobre el valle del río objeto del estudio y donde la cuarcita es mayoritaria, aunque existe una proporción variable, siempre minoritaria, de sílex.

Los yacimientos del primer grupo poseen una fuerte representación macroindustrial en cuarcita, con piezas realizadas con pocos gestos técnicos y presencia escasa, aunque sostenida, de "percutor blando o elástico", casi exclusivamente en los bifaces. La industria de sílex ofrece una escasa representación de útiles sobre lasca y testimonial de macroindustria (sólo presente en seis yacimientos, aunque el grupo bifacial sólo está representado en tres). En el segundo grupo se repiten las características puestas de manifiesto anteriormente con la circunstancia de que en los dos con industria en cuarcita, ésta se muestra minoritaria. Y en el tercer grupo, a su vez, se determinan dos subgrupos en base a las características morfotecnotipólogicas de las industrias, uno (formado por cinco yacimientos) con una fuerte representación macroindustrial en cuarcita y uso, casi testimonial, del sílex; y el otro (con nueve conjuntos industriales), con variable representación de las industrias de sílex, pero siempre minoritaria con respecto a la cuarcita, y con escasa presencia del macroutillaje, siempre en cuarcita, y características similares en los útiles sobre lasca de cuarcita y sílex.

\section{CUCHILLOS DE DORSO NATURAL}

Los cuchillos de dorso natural son uno de los tipos de útiles contenidos en la lista normativa dictada por F. Bordes (1961) que más polémica han suscitado (Moloney, N. 1996) dado que en muchos casos pueden responder a una característica técnica de la talla, generalmente en cuarcita, y no a la necesidad de la fabricación de un útil con esas características. A simple vista es imposible separar los cuchillos de dorso natural que cumplen fielmente las características exigidas de los que no las cumplen en su totalidad aunque ofrezcan un aspecto general propio de ellos: el dorso relativamente inclinado con respecto al plano de la cara bulbar y la falta de huellas de uso ${ }^{1}$. Por ello cada vez son más los autores que expresan sus dudas sobre la necesidad de mantener en la citada lista el útil en cuestión, al menos en los conjuntos industriales realizados en cuarcita, y la mayoría de ellos correspondientes a medios de graveras. En este estado de cosas presentamos una serie de características peculiares de los cuchillos de dorso natural que a lo largo del análisis de las industrias de cuarcita del Bajo Corbones hemos ido detectando y que podrían ayudar a la consideración definitiva de esta pieza como verdadero útil.

1. Según nuestra experiencia el corte de una masa muscular o el de huesos de poco calibre no provoca levantamientos en el filo -melladuras- de una roca tan dura como la cuarcita. Por otro lado, encontrándose en un medio de graveras es muy fácil que la parte más vulnerable de la pieza, el filo, aparezca con variados levantamientos, por lo que puede confundirse con verdaderas huellas de uso. 
El cuchillo de dorso natural es uno de los útiles más comunes en nuestros yacimientos. Definido por Bordes como "Les couteaux à dos naturel sont des éclats ou des lames présentant un tranchant d'un côté et de l'autre une surface de cortex jouant le rôl du dos obtenu par retouche dans les formes précédentes. Ce cortex doit être perpendiculaire ou relativement peu oblique sur le plan d'aplatissement ... mais un tel objet ne peut être compté comme couteaux à dos naturel que s'il présentent des traces d'utilisation sur le tranchant", nuestros cuchillos cumplen las características descritas en buena medida.

No obstante debemos comentar que hemos admitido como tales, por una parte aquellos ejemplares cuyo dorso cortical aparece con cierta inclinación al plano de extracción ${ }^{2}$ y por otra algunos ejemplares sin huellas de uso aparente en el filo. Más adelante nos extenderemos en este punto.

La proporción de cuchillos de dorso natural en relación con el total de útiles sobre lasca en cada subconjunto (cuarcita o sílex dentro del conjunto general que representa un yacimiento) se mantiene de forma más o menos constante en todos y cada uno de los yacimientos en razón del grupo en el que ha sido incluido siendo uno de los útiles más repetidos en nuestro estudio.

En el desarrollo del análisis observamos que de forma reiterativa, entre los 539 cuchillos de dorso natural analizados en cuarcita y los 50 de sílex, aparecían piezas ofreciendo en el dorso un retoque irregular y/o discontinuo. En principio pensamos que podía ser consecuencia del continuo contacto con los elementos que forman parte de la carga de un depósito aluvial. Pero más adelante observamos que en algunos casos el retoque era continuo y sobre todo, demasiado localizado en una parte del dorso (mitad distal) para seguir aceptando irrevocablemente un origen mecánico, aunque mantenía una alta relación de conformaciones irregulares. Como quiera que esta misma circunstancia se daba en el filo del útil en mucha menor proporción, por naturaleza más frágil que la misma arista del dorso y sometido a las mismas condiciones mecánicas, decidimos hacer una observación más intensa sobre esta característica.

Así, comprobamos que todos los subconjuntos de cuarcita ${ }^{3}$ correspondientes a los distintos conjuntos industriales relacionados ofrecían una constante proporción de cuchillos con esta particularidad, cuya media se establece en un $35,18 \%$ del total de los ejemplares analizados, encontrándose dos yacimientos -recuérdese que el número de piezas que forman los diversos conjuntos son muy variados, moviéndose en una banda que va de aquéllos en los que los útiles apenas llegan a la decena, hasta los que superan el centenar- sin presencia del útil en cuestión y ocho (sin incluir los anteriores) en los que no se ha detectado la particularidad comentada (que coinciden con colecciones muy cortas).

Igualmente comprobamos que este retoque es simple mayoritariamente, pero a menudo marginal, rara vez profundo, y en menor medida abrupto o plano. Generalmente es irregular, y en menor proporción discontinuo, y se ofrece único o con escasos golpes y casi siempre directo, difícilmente inverso. En algunos casos, el retoque delimita un denticulado, o aparece bien configurado, continuo, formando un frente de raspador propiamente, o una muesca, o a modo de truncadura o modelando un perforador poco destacado (bec).

Como ya ha quedado apuntado, la mayoría de las piezas presenta la zona del dorso retocada en su mitad distal, mayoritariamente en el tercio distal y en buena proporción correspondiente al extremo, siendo no pocos los localizados en el ángulo laterodistal, aunque se controlaron algunos elementos con un retoque irregular ocupando casi todo el dorso y de forma discontinua (sin poder ser incluidos entre los de dorso atípico). Igualmente se detectaron algunos ejemplares en los que el extremo distal se correspondía con un flanco previo o una rotura intencionada. Teniendo en cuenta que estas circunstancias estaban presentes en piezas que no ofrecían huellas de uso en el filo, que en medio de graveras puede muy bien obtenerse por contacto mecánico, es por lo que, al hilo de lo que exponíamos anteriormente, se han recogido como tales útiles.

2. Vallespí (1979): "cuya colección quizás cabría ampliar con algunas de flancos dorsales corticales o de talla entre las lascas con huellas de uso"

3. En el estudio general de estos yacimientos se analizaron por separado las industrias de cuarcita y sílex. 
Aunque el retoque se hace mucho más patente en los cuchillos realizados en cuarcita que en sílex, de forma general podemos apuntar los siguientes datos:

\begin{tabular}{|c|c|c|}
\hline \multirow{2}{*}{\multicolumn{3}{|c|}{ Retoque }} \\
\hline & & \\
\hline & Ret. simple: & $46,11 \%$ \\
\hline & Ret. simple irreg. & $21,11 \%$ \\
\hline & Ret. marginal: & $17,78 \%$ \\
\hline & Ret. Alternante: & $0,56 \%$ \\
\hline & Ret. abrupto: & $3,33 \%$ \\
\hline & Ret. plano: & $1,67 \%$ \\
\hline & Ret. sobreelevado: & $0,56 \%$ \\
\hline & Ret. bifacial: & $0,56 \%$ \\
\hline & Golpe único: & $5,56 \%$ \\
\hline & Doble golpe: & $1,11 \%$ \\
\hline & Triple golpe: & $1,67 \%$ \\
\hline \multicolumn{3}{|c|}{ Localización } \\
\hline & Extremo distal: & $39,90 \%$ \\
\hline & Tercio distal: & $32,02 \%$ \\
\hline & Mitad distal: & $12,81 \%$ \\
\hline & Mitad proximal: & $1,97 \%$ \\
\hline & Zona medial: & $1,97 \%$ \\
\hline & Todo el dorso: & $11,33 \%$ \\
\hline \multicolumn{3}{|c|}{ Conformación } \\
\hline & Raspador: & 6 ejemplares \\
\hline & Denticulado: & 6 ejemplares \\
\hline & Muesca: & 4 ejemplares \\
\hline & Perforador atíp.: & 2 ejemplares \\
\hline & Truncadura: & 19 ejemplares \\
\hline & Fractura intenc.: & 15 ejemplares \\
\hline
\end{tabular}

Sin desechar la posibilidad, ya comentada, de que en algunos casos el origen del retoque sea fortuito, debemos admitir que una alta proporción de estos retoques debieron producirse intencionadamente. Aunque no hemos encontrado ningún comentario a esta circunstancia en otros estudios ${ }^{4}$, y teniendo en cuenta que en las investigaciones llevadas a cabo en el ámbito del Bajo Guadalquivir (Vallespí y Vallespí et alii 1991 y 1992; Caro 1999) los cuchillos de dorso típicos son escasos, y sin embargo, como ya se ha citado, los de dorso natural pasan a ser útiles punteros en las listas normativas de la mayoría de nuestros yacimientos paleolíticos, parece posible establecer una relación entre la débil presencia de cuchillos de dorso típico y la fuerte de los de dorso natural, pudiendo ser éstos los sustitutos de aquéllos en ambientes de graveras

4. J. A. Caro nos ha comentado, a requerimiento nuestro, que también encuentra estos tipos de retoque en algunos de los cuchillos de dorso natural de su estudio. 
de la zona meridional ${ }^{5}$, y por lo tanto estar sujetos a las variaciones que Bordes hacía notar en aquéllos “... Sous-type achéulen. Dos large, souvent peu abrupt, l'outil se terminant assez fréquemment en pseudograttoir". Así pues es atractivo relacionar este retoque distal en nuestras piezas de dorso natural con el de los de dorso típico del subtipo Achelense definido por Bordes.

No obstante lo argumentado, insistimos en la posibilidad de que una buena parte de este retoque pudiese tener un origen mecánico, y esta hipótesis podría estar soportada en el hecho de que en uno de los yacimientos en superficie sobre los depósitos del Guadalquivir (situados, en nuestra opinión, en posición primaria, y por lo tanto no sometido a transporte) los cuchillos con este retoque son muy escasos $-6,12 \%-$, aunque la relación sube a niveles normales en seis de los yacimientos con semejantes características (en dos no existen, pero el número total de cuchillos es muy corto: cuatro y uno).

En resumen, los cuchillos de dorso natural del río Corbones, muy abundantes como corresponde a yacimientos relacionados con depósitos de cuarcita, se caracterizan en una notable proporción por presentar un retoque irregular en la zona distal, de forma general, y en el ángulo laterodistal en particular, y cuyo objeto queda fuera de nuestras posibilidades, aunque es evidente que en algunas ocasiones podrían jugar un papel funcional inequívoco, actuando la pieza como útil dúplice.

\section{BIFACES DE DORSO NATURAL}

Otra de las particularidades técnicas diferenciadoras que hemos podido detectar en el amplio conjunto de piezas de cuarcita estudiadas en nuestro análisis corresponde a los bifaces de dorso.

Ya hemos comentado que el grupo bifacial está presente en la gran mayoría de los yacimientos, siendo el elemento característico en los correspondientes a los afloramientos de los depósitos del río Corbones del tramo bajo y parte final del tramo medio, y en los cinco que conforman el subgrupo de yacimientos con fuerte presencia macroindustrial de los situados sobre las formaciones del Guadalquivir. No obstante, posee una baja, en muchos casos testimonial o nula, representación en el otro subgrupo del Guadalquivir y en el yacimiento cuyas industrias fueron realizadas en sílex exclusivamente. Así mismo, la inmensa mayoría de los bifaces fueron realizados en cuarcita, siendo escasos los testimonios realizados en sílex.

Dentro del grupo bifacial, el bifaz es la pieza más común en todos los yacimientos donde este grupo está presente. En total se han contabilizado ochocientos ochenta y tres bifaces, de los cuales sólo siete fueron elaborados en sílex En general son útiles fabricados con pocos gestos técnicos, espesos, con base reservada y realizados sobre canto, conociendo en algunos casos la talla de "percutor blando". Atendiendo al sistema propuesto por Gilead (1970), los de aspecto cordiforme son los más repetidos, con más de la mitad del inventario, seguidos a cierta distancia de los de aspecto redondeado, y más alejados de los "otros" y de los de filo transversal y aspecto apuntado, estos dos últimos con una representación semejante. Sin embargo, la presencia de estos tipos es sostenida en la mayoría de los conjuntos, de forma que el que aquí nos ocupa, el de dorso natural, forma parte de un buen número de inventarios. En total se han documentado ochocientos setenta y seis bifaces en cuarcita y siete en sílex. De ellos, cincuenta y siete son de dorso y entre éstos cuarenta son de dorso natural ${ }^{6}$.

Bordes recoge entre los bifaces no clásicos un tipo que denomina "biface à dos", definido dentro del apartado "Bifaces-racloirs" y admitiendo entre ellos el subtipo bifaz con dorso natural: "Nous préféririons

5. Esta circunstancia parece observarse en las investigaciones de la Meseta, tanto en ambientes achelenses como Pinedo, Sartalejo, Galisancho y buena parte de los yacimientos adscritos al círculo de La Maya, como en mesopaleolíticos -Dehesilla y la Parra-)

6. En la nota resumen escrita para la publicación melillense aparecían por error treinta y dos bifaces de dorso natural cuando en realidad la cifra alcanza las cuarenta piezas. 
le terme, plus exact, de biface à dos. Fréquents dans le Micoquien d'Allemagne, de forme général lancéolée ou micoquienne, ils présentent pour la pointe ou la partie médiane une section triangulaire, avec un dos abrupt ou semi-abrupt fait par retouches ou par une utilisation d'un méplat. ..." Aunque no hace comentario alguno a la razón por la cual se encuentra dentro del nombre genérico de bifaz-raedera, mirando las láminas de dibujo observamos que hay un bifaz de dorso natural ( $\mathrm{n}^{\circ} 4$ de la lámina 84 ) clasificado como biface-racloir, que es el que más se asemeja a nuestros bifaces de dorso natural, y en él comprobamos que el borde opuesto al dorso ofrece una retalla cuidada alternante que le da el carácter de raedera. Igualmente, la mitad distal del borde opuesto, es decir, el del dorso, aparece conformada por extracciones medias y pequeñas, presentando también retalla parcial pequeña muy cuidada. Tampoco Merino (1969) hace alusión al carácter de raedera de uno o dos bordes del bifaz de dorso, aunque sí los recoge dentro de ese mismo epígrafe.

Entre los bifaces realizados en cuarcita analizados en nuestro río hemos observado que los de dorso natural se alejan bastante de la definición arriba expresada en cuanto que no presentan el aspecto general de bifaces lanceolados o micoquiense, sino más bien de amigdaloides (todos son espesos) y no ofrecen sección triangular en su punto medio sino ovalar ligeramente achatada en el borde correspondiente al dorso, en correspondencia con el canto soporte. Igualmente presentan el borde opuesto al dorso totalmente tallado (entendiendo hasta la base que normalmente es reservada) y generalmente con retalla más o menos cuidada, aunque esta circunstancia es bastante común en otros conjuntos de medios fluviales.

Con estas características se hizo una primera ordenación de bifaces de dorso natural. Cuando se analizaron por separado, observamos, dentro de este grupo, que había una serie de dieciséis piezas que presentan la particularidad de que uno de los bordes, siempre opuesto al dorso, presenta una retalla generalizada (en dos casos localizada en la zona medial, aunque extendiéndose hacia los extremos), continua, normalmente cuidada, a veces sobreimpuesta, escalariforme, que afecta generalmente al anverso, no participando de estas características el reverso cuyo tratamiento es generalmente somero, con retalla localizada en las intersecciones de las extracciones primarias. El borde opuesto, es decir, el correspondiente a la mitad distal del correspondiente al dorso, no presenta tratamiento alguno complementario.

Igualmente, este borde se halla conformado por el propio dorso que ocupa al menos la mitad basal del borde, y por una única extracción, amplia y profunda, en la parte restante (mitad distal), contándose en algún caso en el que esta gran extracción se hallaba complementada con otra pequeña en su extremo. Igualmente el dorso se corresponde con el borde derecho en una mayoría de ocasiones. Esta última particularidad le da a la pieza una conformación anatómica ideal para ser asida con la mano, sirviendo el hueco de la extracción amplia para colocar el dedo pulgar.

Desde el punto de vista tipológico podrían asociarse a los bifaces raederas, ya descritos por Bordes y recogidos por varios autores" o a los "bifaces à encôche" citados por A. Turq (1992).

Igualmente se han detectado una serie de piezas que participan parcialmente de estas características aunque mantienen las esenciales: piezas sobre canto, talla convergente, tratamiento cuidado en uno de los bordes (talla y retalla) y el otro con mitad basal reservada y distal conformada por un único levantamiento. En oposición a los anteriores, éstos sólo ofrecen talla unifacial, pudiendo en algunos casos incluirse dentro de los unifaces ${ }^{8}$ y en otros entre los cantos tallados de talla desarrollada.

7. J. M. Merino (1969) describe este tipo siguiendo fielmente a Bordes.

P. Utrilla (1986: 36): “... Nos referimos al problema de los bifaces de dorso lateral, muy frecuentes en la Península, los cuales en el sistema Bordes deberían clasificarse como raederas bifaciales. Hemos dudado en poner un tipo específico para ellos pero, dado que el dorso aparece como elemento acumulativo de bifaces con silueta y medidas bien definidas, hemos optado por situarlo al final, clasificando estos bifaces en su tipo morfológico y métrico".

8. Hemos definido como unifaces aquellas piezas sobre canto con talla unifacial desarrollada que ocupa más de las dos terceras partes de la superficie. 


\section{BIBLIOGRAFÍA}

BORDES F. (1961): "Typologie du Paléolithique ancien et moyen”. Edition 1979.

CARO GÓMEZJ.A. (1999): Yacimientos e industrias achelenses en las terrazas fluviales de la Depresión del Bajo Guadalquivir, Tesis doctoral. Facultad de Geografía e Historia de la Universidad Hispalense de Sevilla.

DÍAZ DEL OLMO, F.; VALLESPÍ, E. y BAENA, R. (1992): "Bajo Guadalquivir y afluentes secundarios: Terrazas fluviales y secuencia paleolítica (trabajos 1990)", A.A.A. 1990: 35-39.

DÍAZDEL OLMO, F.; VALLESPÍ, E.; BAENA; R. y ÁLVAREZ, G. (1991): "Bajo Guadalquivir. Terrazas fluviales y secuencia paleolítica", A.A.A. 1989: 17-20.

ENAMORADO, J. (1989): "La Torrecilla y la Parra: Análisis de la industria lítica de dos yacimientos de época paleolítica en el Valle del Manzanares (Madrid)", Boletín del Museo Arqueológico Nacional VII: 9-27.

FERNÁNDEZ CARO, J.J. (1997): "Bifaces y cuchillos de dorso natural. Particularidades técnicas de dos útiles del Paleolítico Inferior y Medio del río Corbones", en Cuaternario del litoral y entorno continental del Mar de Alborán. Resumen de comunicaciones. Melilla: 107-108.

FERNÁNDEZ CARO, J.J. (1998): Las industrias.líticas paleolíticas del Bajo Guadalquivir: el río Corbones Tesis doctoral. Facultad de Filosofía y Letras de Córdoba. Inédita.

FERNÁNDEZ, J.J.; BAENA, R. y GUERRERO, I., (1999): "Poblamiento e industrias líticas a orillas del río Corbones. La Puebla de Cazalla (Sevilla)", Cuadernos de Historia 2. Ayuntamiento de la Púebla de Cazalla.

FERNÁNDEZCARO, J.J. (en prensa): “El Paleolítico Medio de medios fluviales: Yacimientos en superficie de la cuenca del Corbones, afluente del Guadalquivir".

MERINO J.M. (1969): "Tipología Lítica", Munibe. Año. XXI, fasc.1-2-3.

MOLONEY, N. (1996): "The effect of quartzite pebbles on the technology and tipology of Middle Pleistocene lithic assemblages in the Iberian Peninsula" en Non flint stone tools and the Palaeolithic occupation of the Iberian Peninsula, edited by N. Moloney, L. Raposo, y M. Santonja. B.A.R. International Series, 649: 107-119

QUEROL, M.A. y SANTONJA, M. (1979): "El yacimiento achelense de Pinedo (Toledo)" E.A.E. 106. Madrid.

SANTONJA, M. (1985): "El yacimiento achelense de El Sartalejo. (Valle del Alagón, Cáceres). Estudio preliminar", Series de Arqueología Extremeña 2.

SANTONJA, M. y PÉREZ GONZÁLEZ, A. (1984): "Las industrias paleolíticas de la Maya I en su ámbito regional", E.A.E. 135. Madrid.

TURQ, A. (1992): Le Paleolithique inferieur et moyen entre les vallées de la Dordogne et du Lot, Tesis doctoral. Inédita.

UTRILLA, P.; RIOJA, P. y RODANES, J.M. (1986): El Paleolítico en la Rioja: II. El término de CañasCirueña, Zaragoza.

VALLESPÍ, E.; CIUDAD, A. y GARCÍA, R. (1979): Achelense y Musteriense de Porzuna (Ciudad Real). Materiales de superficie, I. (Colección E. Oliver), Museo de Ciudad Real. Colección Estudios y Monografías, 1.

VALLESPÍ, E. (1992): “Las industrias Achelenses de Andalucía: Ordenación y comentarios”, Spal 1:61-78.

VALLESPÍ, E. y DÍAZ DEL OLMO, F. (1990): "Industrias de cuarcitas y comienzos del empleo del sílex en la secuencia del Paleolítico Inferior y Medio del Bajo Guadalquivir", Quarzite and other non-flint lithic material in the Iberian Palaeolithic. B.A.R. Univ. of Oxford. 

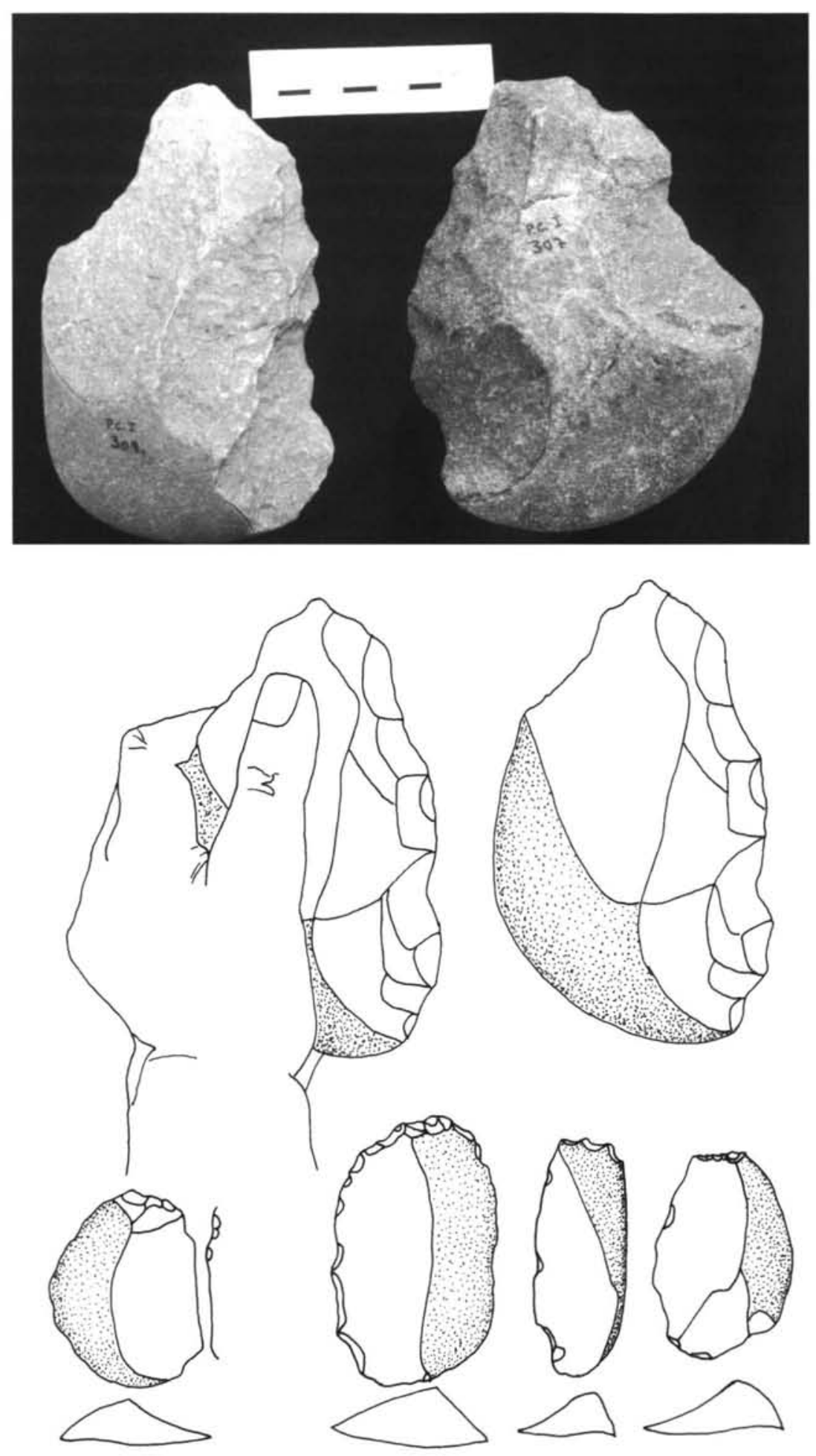

Propuesta de asido de los bifaces de dorso natural. Cuchillos de dorso natural mostrando el retoque distal. 